\title{
Further observations on spermiophagy by murine peritoneal macrophages in vitro
}

\author{
R. Y. Ball, N. Scott and M. J. Mitchinson \\ Department of Pathology, Tennis Court Road, Cambridge CB2 IQP, U.K.
}

\begin{abstract}
Summary. Resident mouse peritoneal macrophages when maintained in cell culture phagocytosed and degraded homologous epididymal spermatozoa. The acrosome and its associated antigens were readily degraded, showing degenerative changes within minutes of spermiophagy and being completely destroyed by $1 \mathrm{~h}$. Other components of the spermatozoon were more resistant and fragments of nuclear material and tail axial filaments could still be recognized within some macrophages up to 7 days after phagocytosis. The findings may be relevant to the disposal of spermatozoa in the genital tract in vivo.
\end{abstract}

\section{Introduction}

Spermiophagy by macrophages is seen within the obstructed male genital tract in lesions occurring naturally (Mitchinson, Sherman \& Stainer-Smith, 1975; Holstein, 1978) and after vasectomy (Alexander \& Tung, 1979; Tung \& Alexander, 1980; Flickinger, 1982). It also occurs in spermatic granulomata (Glassy \& Mostofi, 1956) and in regional lymph nodes after vasectomy in man (Ball, Naylor \& Mitchinson, 1982) and domestic animals (Ball \& Setchell, 1983). Spermiophagy may be a means of disposing of trapped or extravasated spermatozoa, and of degrading them, thus preventing the development of sperm autoallergy. Alternatively, the macrophage may process and present phagocytosed sperm antigens so that they are more likely to induce autosensitization, as is known in many experimental systems (Humphrey, 1982).

Ball \& Mitchinson (1977) have previously shown that mouse spermatozoa are degraded by macrophages to ceroid pigment within a few days, but the relevance of sperm degradation to antigenicity prompted a study of the earlier events in this process.

\section{Materials and Methods}

Male BALB/c mice aged 2-3 months were killed by cervical dislocation and peritoneal cells were obtained by intraperitoneal injection of $3 \mathrm{ml}$ Hanks balanced salt solution (HBSS: Flow Laboratories, Irvine, U.K.) followed by gentle abdominal massage and aspiration. Pooled peritoneal aspirates were dispensed in 1-2 ml samples to tissue culture dishes $(35 \times 10 \mathrm{~mm}$ : Falcon Plastics, Oxnard, CA, U.S.A.) which contained sterile $22 \mathrm{~mm}^{2}$ glass coverslips. The suspensions were incubated at $37^{\circ} \mathrm{C}$ for $45-60 \mathrm{~min}$ to allow the macrophages to adhere, then vigorously washed twice with HBSS to remove non-adherent cells. The HBSS was replaced by $1 \mathrm{ml}$ samples of culture medium (Ball \& Mitchinson, 1977) and the cells were incubated overnight at $37^{\circ} \mathrm{C}$ in an atmosphere of $5 \% \mathrm{CO}_{2}$ in air. 
Immunofluorescence microscopy. After overnight incubation, the culture medium was replaced by one containing homologous spermatozoa $\left(10-25 \times 10^{6} / \mathrm{ml}\right)$ obtained immediately beforehand by flushing the lumina of the deferent ducts and caudae of the epididymides of several mice with culture medium using a syringe and 25 -gauge needle. The cultures were incubated at $37^{\circ} \mathrm{C}$ in the $\mathrm{CO}_{2}$-enriched atmosphere and were examined for the presence of sperm antigens by indirect immunofluorescence (IIF) at 1, 5, 10, 20,60 and 120 min and 4, 10, 22 and $34 \mathrm{~h}$. Coverslips were washed twice in phosphate-buffered saline (PBS), dried under a fan and fixed in methanol for 30 min. The first antibody was $1: 50$ rabbit anti-BALB/c epididymal sperm serum (titre $1: 4096$ ) which had previously been absorbed several times with extracts of $B A L B / c$ liver, spleen and kidney. Prior treatment of the specific antibody with methanol-fixed $B A L B / c$ spermatozoa abolished IIF staining. The labelled antibody was $1: 20$ fluorescein isothiocyanate(FITC)-conjugated swine antirabbit immunoglobulin (Dako, Copenhagen, Denmark). All dilutions were in PBS. The coverslips were examined with a Zeiss fluorescence microscope adjusted for FITC. The experiment was performed 5 times.

Scanning electron microscopy. After overnight incubation, spermatozoa were added as above and the cultures incubated for $15 \mathrm{~min}$. Some cultures were then washed and fixed but others were washed once with HBSS and incubated for an additional 15-45 min in fresh medium before processing for scanning electron microscopy. Before fixation, the coverslips were washed 7 times in HBSS at $37^{\circ} \mathrm{C}$. The cultures were then fixed for $1 \mathrm{~h}$ at $4^{\circ} \mathrm{C}$ using $2 \%$ glutaraldehyde $+1 \%$ paraformaldehyde in phosphate buffer, $\mathrm{pH} 7 \cdot 2$. The coverslips were washed 3 times with PBS, post-fixed in $1 \%$ osmium tetroxide for $2 \mathrm{~h}$ at $4{ }^{\circ} \mathrm{C}$, thoroughly washed in PBS, dehydrated in acetone and $\mathrm{CO}_{2}$ critical-point dried using a Polaron E3000 drier (Polaron, Watford, Herts, U.K.). The coverslips were cut into segments up to $5 \mathrm{~mm}$ across and samples were mounted on stubs and coated in gold, $30 \mathrm{~nm}$ thick, using a Polaron E5000 spattering unit. Specimens were viewed in a Stereoscan S600 electron microscope (Cambridge Instruments, Cambridge, U.K.).

Transmission electron microscopy. The macrophages were allowed to adhere to the bottom of the tissue culture dishes rather than to glass coverslips. Conditions of culture were otherwise identical.

After overnight incubation, the medium was changed (control dishes) or replaced by medium containing spermatozoa (experimental dishes). All experimental dishes, except those fixed 5 and 10 min after the addition of the sperm suspension, were washed thoroughly with HBSS after 15 min to remove free spermatozoa. Several experimental dishes were fixed after the addition of spermatozoa at $5,10,15,30,45$ and $60 \mathrm{~min}, 2,3,4,6,12,15$ and $24 \mathrm{~h}$, and at daily intervals up to 6 days. Control dishes were fixed daily for 7 days. Before fixation, the cultures were washed with PBS, fixed in $3 \%$

\section{PLATE 1}

Fig. 1. Initial attachment of BALB/c spermatozoa to an homologous resident peritoneal macrophage in vitro. One spermatozoon (arrow) has attached by the tip of the acrosome, where small pseudopodia are already developing. The other spermatozoon has attached by the convex surface of the acrosome. Both acrosomes are intact. Note the mitochondrial sheath of the midpieces. SEM, $\times 3750$.

Fig. 2. Initial attachment of a murine spermatozoon to an homologous resident peritoneal macrophage in vitro. The sperm surface over the acrosome is closely related to the plasmalemma of the macrophage but a tiny gap exists between the two cells. TEM, $\times 56000$.

Fig. 3. Murine peritoneal macrophage in vitro. One spermatozoon has been partly phagocytosed. A faint remnant of acrosomal staining (arrow) is apparent. The portion of the midpiece which has been phagocytosed has lost its IIF staining. Sperm components outside the macrophage continue to show bright fluorescence. IIF, $\times 1600$.

Fig. 4. Murine spermatozoa stained by the IIF technique. Note the bright staining of the acrosome and the 'tramline' appearance of the midpiece and tail. IIF, $\times 1600$. 
PLATE I
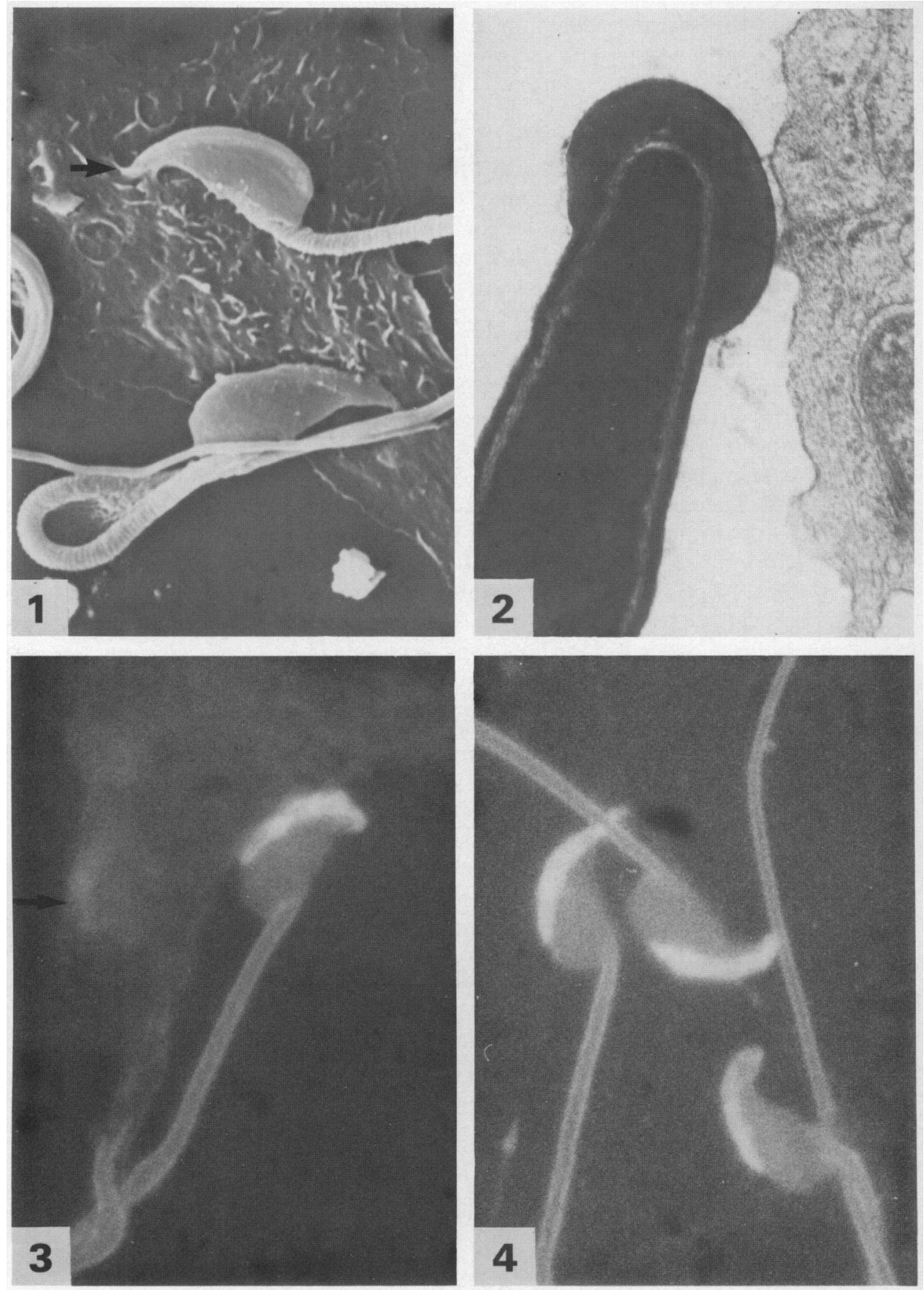

(Facing p. 222) 
PLATE 2
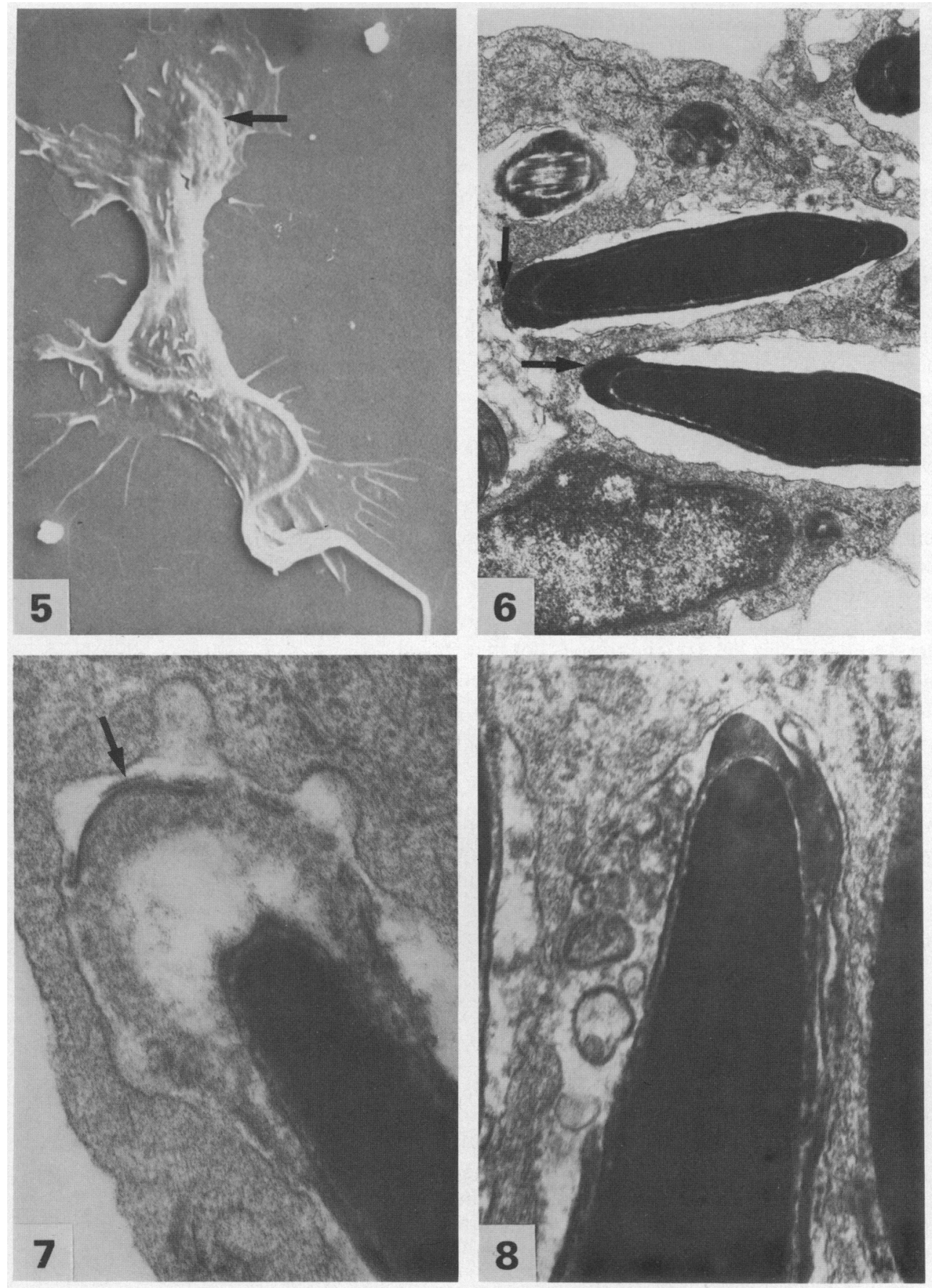
glutaraldehyde in $0 \cdot 13 \mathrm{M}$-cacodylate buffer for $1 \mathrm{~h}$ at room temperature, and the cells suspended using a silicone rubber 'policeman'. The suspension was then centrifuged at $500 \mathrm{~g}$ for 5-10 min, the supernatant discarded and the pellet washed in $0.2 \mathrm{M}$-cacodylate buffer. Specimens were post-fixed in $2 \% \mathrm{OsO}_{4}$ for $1-2 \mathrm{~h}$, dehydrated in a graded series of alcohols and in propylene oxide, and embedded in Spurr resin. Sections were cut and stained with uranyl acetate and lead citrate and examined with a Philips EM 300 transmission electron microscope.

\section{Results}

The interaction between mouse peritoneal macrophages and homologous spermatozoa in vitro was composed of attachment of the spermatozoa to the macrophage surface; phagocytosis; and degradation.

\section{Attachment}

The initial attachment of the spermatozoa to the macrophage surface occurred within seconds of their addition to the cultures and was almost always by the head, generally by the hooked tip but occasionally by the convex surface (Pl. 1, Fig. 1). The membranes of the two cells were closely apposed with a narrow, electron-lucent gap between them (P1. 1, Fig. 2). Spermatozoa rarely adhered by the midpiece, but when they did it was most often in the region of the cytoplasmic droplet. IIF staining of attached, but not yet phagocytosed, spermatozoa showed bright fluorescence of the acrosome and of the surface of the midpiece and tail (Pl. 1, Fig. 3) identical to that seen in smears of spermatozoa (Pl. 1, Fig. 4).

\section{Phagocytosis}

Within a few minutes of attachment the macrophage extended pseudopodia over the surface of the sperm head and along the midpiece and mainpiece of the tail. Such pseudopodia were closely related to the sperm surface and often coiled around the tail several times to produce a spiral in transverse section. In other cells, spermatozoa disappeared into deep phagocytic clefts. Examination of living preparations by phase-contrast microscopy showed persistent beating of the

\section{PLATE 2}

Fig. 5. Cultured murine peritoneal macrophage which has almost completely phagocytosed one spermatozoon. The sperm head (arrow) and the tail, which has adopted a sinuous course, are readily apparent inside the phagocyte. SEM, $\times 1600$.

Fig. 6. Two spermatozoa are present within separate, capacious phagocytic vacuoles in a cultured peritoneal macrophage. The initial points of attachment between the spermatozoa and plasma membrane, now internalized, persist (arrows). The acrosomes appear intact. Sections of tail are present in other phagosomes at the edges of the figure. TEM, $\times 20000$.

Fig. 7. A mouse sperm head lies in a macrophage phagosome. The sperm nucleus and inner acrosomal membrane appear intact but the acrosome is degenerating. The outer acrosomal and plasma membranes persist in some places (arrow) but in others are absent. The acrosome is swollen and it is becoming electron-lucent, probably as a result of loss of its contents. TEM, $\times 65000$.

Fig. 8. Further degeneration of a murine sperm head after phagocytosis. The nucleus, inner acrosomal membrane and perforatorium appear unaffected but the acrosome has been destroyed and is replaced by a mass of membranous vesicles lying within the phagosome. TEM, $\times 65000$. 
projecting tail many minutes after the head had been phagocytosed. Even within 5 min of the addition of the sperm suspension to the macrophage culture, some sperm heads and midpieces had been engulfed and by $1 \mathrm{~h}$ many spermatozoa were almost completely phagocytosed. Their outlines were easily seen by scanning electron microscopy and were evident as loops or serpentine curves below the macrophage surface (Pl. 2, Fig. 5). By 3 h some macrophages had phagocytosed up to 8 spermatozoa but, despite this, the phagocyte maintained a normal shape; the phagocytosed spermatozoa were forced to adapt to the confines of their new environment, rather than vice versa. Inside the macrophage, the sperm head lay in a capacious phagocytic vacuole. The initial attachment of the spermatozoon to the cell membrane persisted within the phagosome (Pl. 2, Fig. 6) and the latter was often seen to be in free communication with the extracellular medium. Fusion of sperm surface membranes to those of the macrophage was never observed; sperm components remained isolated inside phagocytic vacuoles.

\section{Degradation}

Phagocytosed spermatozoa were degraded in a characteristic manner. Between 5 and 10 min after spermiophagy, localized regions of the plasma and outer acrosomal membranes appeared to become detached and coiled, forming vesicles. Such changes affected only limited segments of the membranes and the underlying acrosome appeared to remain intact. At the same time, IIF showed patchy, focal loss of acrosomal staining. By $30 \mathrm{~min}$ acrosomal changes were conspicuous, with swelling, focal loss of the external membranes and electron-lucency of the interior, suggesting the leaching of its contents (Pl. 2, Fig. 7). Rarely, membrane-bound vesicles were seen within the acrosomal matrix. By $1 \mathrm{~h}$ acrosomal degeneration was pronounced with a residual mass of membrane-bound, vesicular structures (PI. 2, Fig. 8). Acrosomal immunofluorescence was severely reduced or abolished by this time (Pl. 1, Fig. 3). Within 2-3 h most of the spermatozoa had lost their acrosomes and acrosomal immunofluorescence but the nucleus, inner acrosomal membrane and perforatorium appeared intact. Over the course of the first $4 \mathrm{~h}$, as they were phagocytosed, the midpiece and tail lost their surface IIF staining but portions of spermatozoa still outside the macrophage showed persistent immunofluorescence.

While the acrosome and plasma membrane appeared to be degraded easily, other sperm components were more resistant. The nucleus showed degenerative changes by $12 \mathrm{~h}$. Its outline became irregular, indicating piecemeal destruction from the periphery. Such changes continued over the next few days but, even after 6 days, degenerate nuclear remnants could still be recognized within some macrophages. The degeneration of the tails also pursued a slow time-course. By $12 \mathrm{~h}$ the mitochondria of the midpiece were swollen and degenerate, demonstrating a loss of cristae and matrix but persistence of their outer membranes. Up to 7 days there was increasing degeneration of the axial filament complex and outer dense fibres. Degenerate tail remnants persisted within macrophages for at least 1 week. As the spermatozoa degenerated, myelin figures and ceroid pigment granules were noted within some macrophages. Large droplets of cytoplasmic lipid accumulated after several days but this was also noted in control preparations.

\section{Discussion}

The present results suggest that macrophages could act on phagocytosed spermatozoa to degrade their antigens, thereby inhibiting an autoallergic reaction.

No special treatment of the macrophages is necessary to allow motile homologous spermatozoa to become attached to their surfaces. Incubation of the macrophages with $0.05 \%$ beef pancreatic trypsin impairs sperm binding, as does the absence of divalent cations. On the other hand, prior treatment of the macrophage cultures with antimouse sperm antiserum improves the binding of 
spermatozoa (Ball, 1983). This is due, presumably, to cytophilic binding of appropriate antibodies to surface Fc receptors. The ease with which murine spermatozoa adhere to homologous macrophages contrasts with the results obtained using cells from guinea-pigs, for which spermiophagy occurs in the presence of serum from animals with experimental allergic orchitis but not when normal control sera are used (Mazzolli, Barrera \& Vilar, 1979). Quite apart from the possibility of species variation, differences of method make direct comparison between the two systems difficult. In a preliminary experiment in which washed human ejaculated spermatozoa were added to a culture of human macrophages obtained as monocytes $24 \mathrm{~h}$ earlier, none of the spermatozoa adhered to the phagocytes, despite persistent motility for several hours (R. Y. Ball, unpublished observation). It seems, therefore, that the human macrophage-sperm interaction may be more complex than in the mouse.

Once phagocytosed, the mouse spermatozoa were degraded in a characteristic way. Acrosome changes occurred rapidly and consisted of vesiculation, swelling and dispersion of the contents. These changes are similar to those taking place during the acrosome reaction, a process occurring before the fertilization of the ovum (Barros, Bedford, Franklin \& Austin, 1967; Austin, 1975). The acrosome reaction involves sperm survival but similar alterations also develop in the acrosome after spermatozoa are exposed to various noxious agents (Austin, 1975). It is probable that the 'acrosomal reaction' observed after spermiophagy is a non-specific response to injury, presumably caused by the macrophage's lysosomal enzymes and oxidative killing mechanisms (Babior, 1978; Weiss \& LoBuglio, 1982). The latter may be particularly effective at inducing a false acrosome reaction because peroxidation of ram spermatozoa in vitro causes damage, especially to the acrosome (Jones \& Mann, 1977). The relative resistance of the nucleus, outer mitochondrial membrane, and of the filaments and fibres of the tail and its fibrous sheath may be because of the disulphide cross-linked keratinoid proteins they contain (Mann \& Lutwak-Mann, 1981). Such indigestible material is likely to be important in the development of the granulomatous reaction to extravasated spermatozoa in vivo (Ball, 1984).

The most important sperm antigens are on the sperm surface. As the surface membrane, especially that of the acrosome, is rapidly destroyed after spermiophagy, it might be that these antigens are quickly rendered innocuous. The rapid loss of IIF staining of the acrosome, midpiece and tail as they were phagocytosed is not likely to be due to antigenic inaccessibility. Methanol fixation would allow the antiserum to penetrate the macrophage and the phagocytic vacuole was shown by electron microscopy initially to be in free communication with the extracellular medium. Moreover, the patchy loss of acrosomal IIF corresponded in timing and appearance with the early acrosomal changes as revealed by transmission electron microscopy. The loss of IIF reflects the disruption of the sperm surface as a whole. Which of the antigens are important in terms of allergic effects in the male is not known and the fate of such antigens within the macrophage is not clear. If macrophages are important in processing sperm antigens for an autoallergic reaction, then some such antigens must be preserved and should, in theory, be detectable by more sensitive techniques.

Macrophages are conspicuous in lesions of the male genital tract and may, as a result of spermiophagy, be intimately related to the development, or prevention of, an autoallergic response to spermatozoa. A necropsy study of men suggests that spermiophagy by macrophages within the genital tract is common and not related to the development of antisperm autoallergy as detected by the tray agglutination test (Ball \& Mitchinson, 1984). Rapid destruction of the sperm surface and its antigens after local phagocytosis would be one means of preventing autosensitization. If so, sperm autoantibodies might only develop after unphagocytosed spermatozoa reach local lymph nodes (Ball et al., 1982; Ball \& Setchell, 1983). The sperm-macrophage interaction, particularly in man, therefore warrants further investigation.

R.Y.B. is funded by an Elmore Medical Research Studentship and St John's College, Cambridge. We thank Miss Louise Walker for help with the SEM, Miss Wendy Gillson for technical assistance and Mrs Helen Wilson for typing. 


\section{References}

Alexander, N.J. \& Tung, K.S.K. (1979) Vasectomy in the rabbit: immunological and morphological effects. In Vasectomy. Immunologic and Pathophysiologic Effects in Animals and Man, ch. 17, pp. 355-377. Eds I. H. Lepow \& R. Crozier. Academic Press, New York.

Austin, C.R. (1975) Membrane fusion events in fertilization. J. Reprod. Fert. 44, 155-166.

Babior, B.M. (1978) Oxygen-dependent microbial killing by phagocytes. New Engl. J. Med. 298, 659-668.

Ball, R.Y. (1983) Aspects of obstruction of the male genital tract in mammals. Ph.D. thesis, University of Cambridge.

Ball, R.Y. (1984) Experimental production of spermatic granulomata in Balb/c mice. Andrologia (in press).

Ball, R.Y. \& Mitchinson, M.J. (1977) Degradation of phagocytosed spermatozoa by mouse peritoneal macrophages in vitro. J. Reprod. Fert. 49, 409-411.

Ball, R.Y. \& Mitchinson, M.J. (1984) Obstructive lesions of the genital tract in men. J. Reprod. Fert. 70, 667673.

Ball, R.Y. \& Setchell, B.P. (1983) The passage of spermatozoa to regional lymph nodes in testicular lymph following vasectomy in rams and boars. $J$. Reprod. Fert. 68, 145-153.

Ball, R.Y., Naylor, C.P.E. \& Mitchinson, M.J. (1982) Spermatozoa in an abdominal lymph node after vasectomy in a man. $J$. Reprod. Fert. 66, 715-716.

Barros, C., Bedford, J.M., Franklin, L.E. \& Austin, C.R. (1967) Membrane vesiculation as a feature of the mammalian acrosome reaction. J. Cell Biol. 34, C1C5.
Flickinger, C.J. (1982) The fate of sperm after vasectomy in the hamster. Anat. Rec. 202, 231-239.

Glassy, F.J. \& Mostofi, F.K. (1956) Spermatic granulomas of the epididymis. Am. J. clin. Path. 26, 13031313.

Holstein, A.-F. (1978) Spermatophagy in the seminiferous tubules and excurrent ducts of the testis in rhesus monkey and in man. Andrologia 10, 331-352.

Humphrey, J.H. (1982) The fate of antigens. In Clinical Aspects of Immunology, 4th edn, ch. 7, pp. 161-186. Eds P. J. Lachmann \& D. K. Peters. Blackwell, Oxford.

Jones, R. \& Mann, T. (1977) Damage to ram spermatozoa by peroxidation of endogenous phospholipids. $J$. Reprod. Fert. 50, 261-268.

Mann, T. \& Lutwak-Mann, C. (1981) Male Reproductive Function and Semen. Springer-Verlag, Berlin.

Mazzolli, A.B., Barrera, C. \& Vilar, O. (1979) Ultrastructural study of the macrophage-cytophilic antibodyspermatozoa complex. Int. J. Androl. 2, 230-240.

Mitchinson, M.J., Sherman, K.P. \& Stainer-Smith, A.M. (1975) Brown patches in the epididymis. J. Path. 115, 57-62.

Tung, K.S.K. \& Alexander, N.J. (1980) Monocytic orchitis and aspermatogenesis in normal and vasectomized rhesus monkeys (Macacca mulatta). Am. J. Path. 101, 17-30.

Weiss, S.J. \& LoBuglio, A.F. (1982) Phagocyte generated oxygen metabolites and cellular injury. Lab. Invest. 47, 5-18.

Received 16 September 1983 